\title{
Simultaneous Localization and Mapping - Gasteditorial
}

\section{Udo Frese}

Online publiziert: 6. Mai 2010

(C) Springer-Verlag 2010

\section{Liebe Leserinnen, liebe Leser,}

Simultaneous Localization and Mapping ist ein Problem mit einem sperrigen Namen - aber einer griffigen Abkürzung: SLAM, wie in Grand Slam, Slam Dunk und Poetry Slam. Also ein Problem, das die Herausforderung schon in der Bezeichnung mit sich führt.

In der Tat, es geht um nichts weniger, als dass ein Roboter sich in einer Umgebung bewegt und dabei ein inneres Abbild der Umgebung, eine Karte, aufbaut. Man kann mit Fug und Recht sagen, dass dies die grundlegendste Intelligenzleistung ist, die sich bezüglich mobiler Robotik stellt. SLAM ist schwierig, weil es ein "Henne und Ei"-Problem ist: Wüsste man, wo jeweils der Roboter ist, wäre es nicht schwer, die einzelnen Wahrnehmungen der Umgebung zu einem Ganzen zusammenzusetzen (Mapping), kennte man die Umgebung, wäre es nicht schwer, den Roboter zu lokalisieren (Localization). Erst beides zusammen, also SLAM, ist die Herausforderung.

SLAM wird nun seit etwas über 20 Jahren untersucht und ich hatte das Glück, gut die Hälfte davon mitzuerleben. Deshalb möchte ich den Herausgebern der KI für die Gelegenheit danken, mit diesem Themenheft einen Moment innezuhalten und sich zu fragen: Wo stehen wir?

U. Frese ( $\square)$

Enrique-Schmidt-Str. 5, 28359 Bremen, Deutchland

e-mail: udo.frese@dfki.de
In dem Überblicksartikel tue ich dies mit der Fragestellung "Welcher Teil von SLAM ist so ausgereift, dass man ihn außerhalb von SLAM benutzen kann?" Kretzschmar et al. hingegen betrachten einen Teil von SLAM, der noch der Lösung harrt, nämlich wie die Karte im Roboter sich langfristigen Änderungen der Umgebung anpassen kann. Mair et al. schieben die Grenze von visuellem SLAM Richtung schneller Bewegungen weiter hinaus, während Sünderhauf et al. und Schwender et al. den Vergleich zu Tieren ziehen, die dies Problem ja seit Jahrmillionen gelöst haben. Hertzberg et al. und Birk et al. gehen in die dritte Dimension, wobei sie besonders unregelmäßige Strukturen interessieren. Eine wichtige Anwendung sind vom Erdbeben zerstörte Gebäude. Zum Schluss frage ich in einem Streitgespräch Sebastian Thrun und José Neira ob SLAM bereits gelöst ist. Die Antwort lautet: Ja, aber irgendwie doch wieder nicht. Es bleibt also spannend. Herzlichst, Ihr

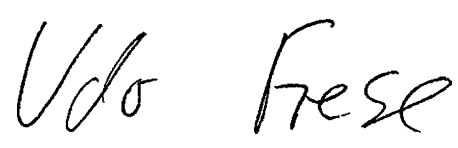

29. April 2010

Udo Frese 